\title{
influence de la taille des grains et de l'altération des roches sur les mesures de l'atténuation des ondes ultrasonores en laboratoire
}

\author{
ultrasonic attenuation testing in rock samples: \\ influence of grain size and of weathering in granites
}

\author{
N. KLIMIS \\ Docteur en Géologie de l'Ingénieur, Thessalonique * \\ J.-.L DURVILLE \\ Chef de la Section Géologie et Matériaux, L.C.P.C. ** \\ J. EHRLICH \\ Ingénieur, Section Mesures-Electronique, L.C.P.C. ${ }^{* *}$
}

Rev. Franç. Geotech. n 47 , pp. $29-38$ (avril 1989)

\section{Résumé}

On présente une méthode de mesure en laboratoire, sur éprouvette de roche "sèche", de l'atténuation des ondes longitudinales. La mesure s'effectue par transmission, à l'aide de transducteurs piézo-électriques; le signal reçu est étudié soit dans sa forme temporelle, soit après transformée de Fourier (en particulier la méthode du rapport de spectres est utilisée). On étudie l'atténuation sur deux séries de roches:

- une série de type granitique, oủ l'on vérifie la forte dépendance de l'atténuation avec la taille des grains de la roche, du fait de la diffraction des ondes,

- une série de trois états d'altération d'une aplite, qui montre la sensibilité du coefficient d'atténuation à la "qualité * de la roche.

II est envisageable que la mesure de l'atténuation puisse compléter l'information apportée par l'indice de continuité pratiqué couramment.

\section{Abstract}

An ultrasonic pulse transmission technique has been developed for measuring
attenuation in idry rocks. Piezo-electric transducers produce P-waves at $0.5,1.0$
or $2.25 \mathrm{MHz}$. The pulse rise time method, and Fourier spectral ratios are used.
Measurements have been made:
- with granitic rocks, each with homogeneous grain, varying in grain diameter
from 0.1 to $5 \mathrm{~mm}:$ a strong dependance of attenuation, due to grain scattering, is
shown,
- with an aplitic rock at three states of weathering: the corresponding quality
factor $Q$ decreases with increasing weathering.
It seems that the proposed measurement of attenuation may supplement the
information given by the Continuity Index (issued from the much easier velocity
measurement).

- Université de Thessalonique, Grèce.

*. LC.P.C. 58, bd Lefebure, 75732 Paris Cedex 15.

Tél. : 48.56 .50 .00 . 


\section{INTRODUCTION}

Dans le domaine de la mécanique des roches et des granulats, la mesure de la vitesse ultrasonore par transmission à travers un échantillon de roche, est pratiquée couramment : cette mesure, très rapide, fournit un élé. ment d'identification du matériau, susceptible de renseigner sur son état d'altération, de microfissura. tion, etc. par l'intermédiaire de l'indice de continuité I.C. $=V_{\text {mes }} / V_{\text {calc }}$ (TOURENQ et al., 1971).

On présente ci-dessous quelques résultats d'une recherche, dont l'objectif est d'exploiter non seulement le temps d'arrivée du signal, mais également la modification du signal lors de la traversée de l'échantillon; en particulier l'atténuation des ondes dans le matériau est évaluée par la méthode du rapport des spectres.

\section{DESCRIPTION DE L'ESSAI}

La chaîne de mesures permet l'étude de l'atténuation des ondes longitudinales, après traversée d'une éprouvette cylindrique de roche « sèche „(KLIMIS, 1987).

\subsection{Le matériel}

Le bâti-support (figure 1) assure une mise en place précise de l'échantillon; une légère charge statique, voisine de $0,2 \mathrm{MPa}$, est appliquée, ce qui améliore la qualité des couplages (couplant utilisé: graisse de vaseline).



Fig. 1. - Bati-support servant aux mesures d'atténuation. Fig. 1. - Experimental apparatus for attenuation measurement.
L'impulsion, dont le temps de montée est inférieur à 10 nanosecondes, est fournie par un générateur Panametrics 5055 PR. Trois couples de transducteurs piézo-électriques (émetteur et récepteur) peuvent être utilisés, correspondant à des fréquences centrales de $500 \mathrm{kHz}$ (bande passante à $-6 \mathrm{~dB}: 250-800 \mathrm{kHz}$ ), $1 \mathrm{MHz}$ (bande passante: $0,5-1,6 \mathrm{MHz}$ ) et $2,25 \mathrm{MHz}$ (bande passante: $1,2-3,2 \mathrm{MHz}$ ).

Le signal est recueilli dans un oscilloscope digital Tektronix, avec une fréquence d'échantillonnage de $25 \mathrm{MHz}$, puis il est traité sur micro-ordinateur Apple Ile.

\subsection{La procédure d'essai et sa fiabilité}

Il est connu que l'atténuation est très délicate à mesurer. que ce soit in situ ou en laboratoire. Pour obtenir une bonne répétabilité de la mesure (figure 2), on a pris un certain nombre de précautions:

- charge statique de 0,2 MPa s'exerçant sur les transducteurs de l'éprouvette;

- éprouvettes dont les faces sont rectifiées;

- temps d'attente de 5 minutes entre la mise en place de l'éprouvette et la mesure, de façon que la graisse de vaseline ait le temps de s'étaler uniformément;

- exclusion des matériaux trop poreux: il semble que la vaseline percole progressivement dans la masse, ce qui peut modifier le signal, pour les roches de porosité supérieure à $25 \%$ environ;

- contrôle de la teneur en eau de l'éprouvette: une longue exposition à l'air ambiant d'un matériau initialement séché à $105^{\circ} \mathrm{C}$ peut conduire à une adsorption d'eau et à un changement sensible de l'atténuation (BOURBIÉ et al., 1986).

Par ailleurs, dans la mesure où c'est l'atténuation que l'on cherche à mettre en évidence, on souhaite que le signal reçu reflète au maximum cette caractéristique:

- la forme de l'éprouvette $(\varnothing=100 \mathrm{~mm}, \mathrm{~L}=$ $66 \mathrm{~mm}$ ) a été choisie de façon que les premières alternances ne solent pas perturbées par les réflexions latérales ;

- l'amplitude du signal recueilli est influencée par le couple transducteur-roche : forme du front d'onde émis leffet de diffraction à partir de la source $\varnothing=$ $25,4 \mathrm{~mm}$ ), facteur de transmission (lié au contraste des impédances acoustiques). Les corrections correspondantes, très complexes, n'ont pas été effectuées.

D'autre part, la réponse des capteurs est influencée par les propriétés du matériau en contact; si l'on était amené à étudier des matériaux très contrastés, l'utilisation de tampons intermédiaires s'imposerait (WINK. LER et PLONA, 1982).

En définitive, on dispose d'un essai d'une bonne fiabilité. Les amplitudes du signal fourni dépendent à la fois de l'atténuation et des propriétés élastiques du matériau $\left(\rho, V_{p}, V_{s}\right)$; ses caractéristiques fréquentielles en revanche semblent être plus représentatives de l'atténuation seule. 


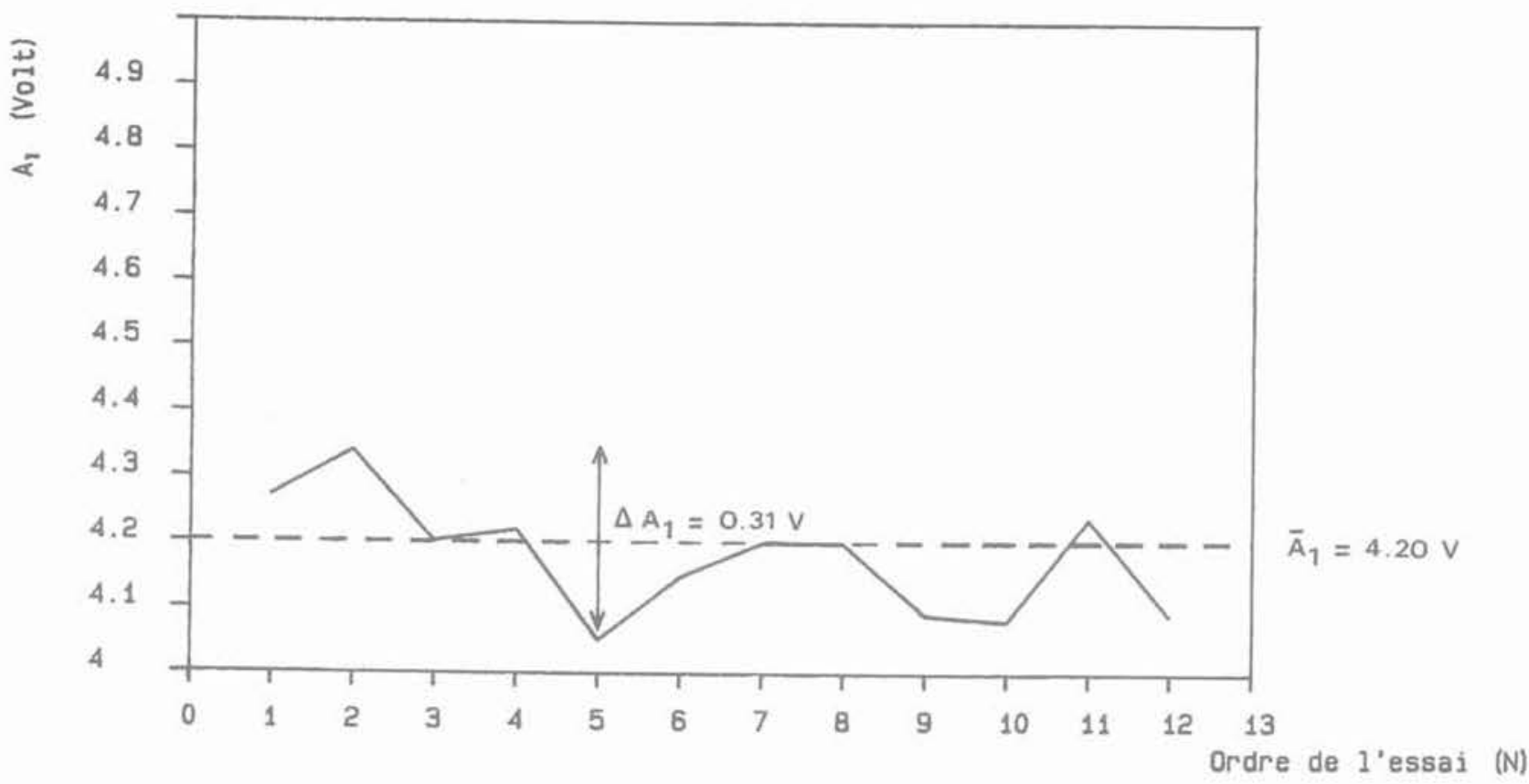

- AMPLITUDE-

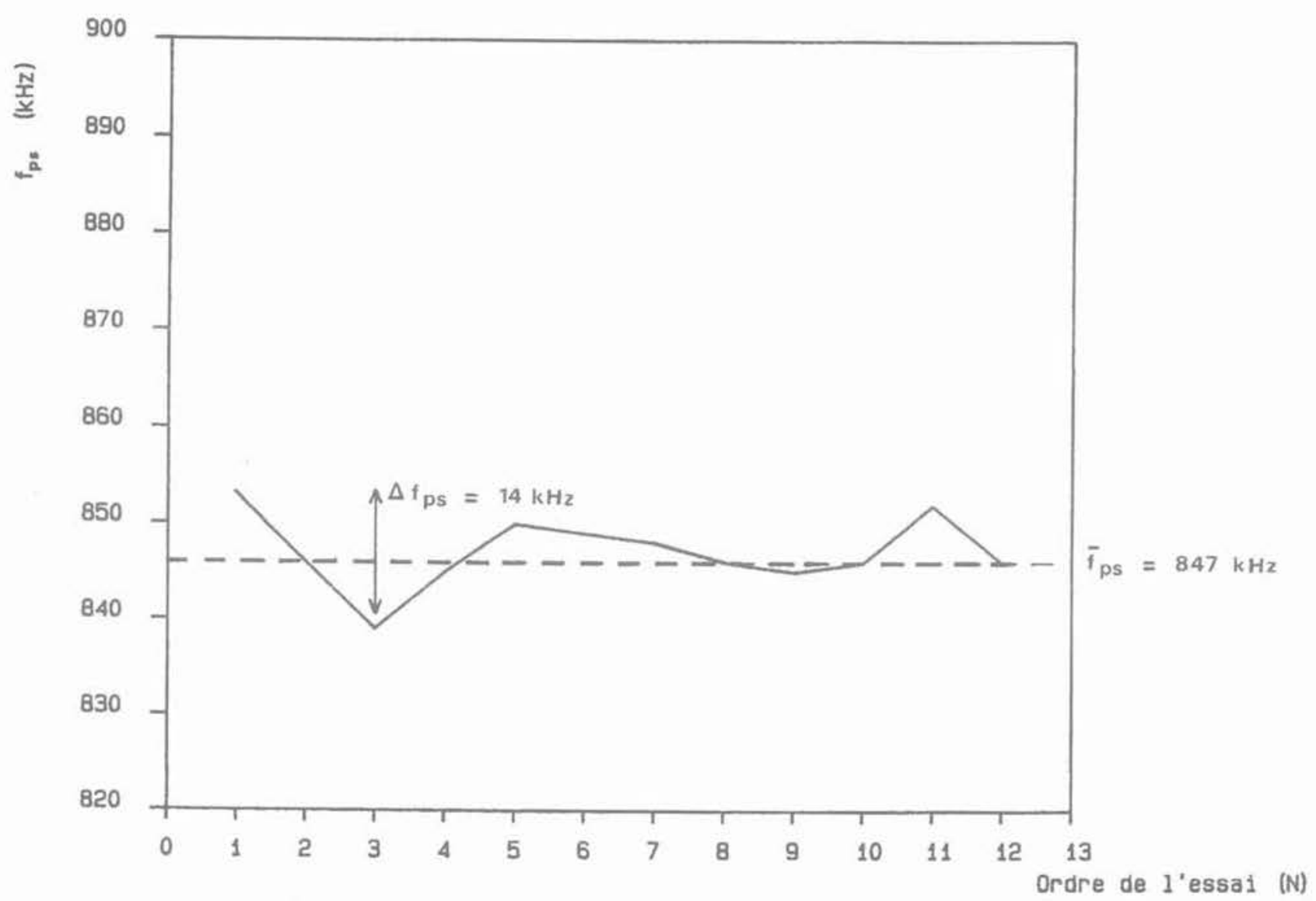

- PSEUDOFREQUENCE-

Fig. 2. - Répétabilité de la mesure: cas de deux paramètres définissant le signal temporel (cf. figure 3), mesurés lors de douze essais indépendants.
Fig. 2. - Repeatability of the amplitude and of the pseudo-frequence measured on the signal received (twelve tests). 


\section{EXPLOITATION DES MESURES}

L'analyse du signal est effectuée :

- dans le domaine temporel: la morphologie des premières alternances est caractérisée par quelques pa. ramètres simples (figure 3 ) : pseudofréquence, temps de montée, amplitude de la première alternance,

- dans le domaine spectral (transformation de Fourier rapide): le module de la transformée de Fourier du signal peut également être décrit par quelques paramètres (fréquence du maximum par exemple); la mé. thode qui sera développée ci-dessous est celle du rapport de spectres (cf. TOKSÖZ et al., 1979). Celle-ci consiste à évaluer le pouvoir atténuant d'un matériau en comparant les signaux obtenus, dans les mêmes conditions expérimentales, avec la roche d'une part, et avec un matériau de référence d'autre part.

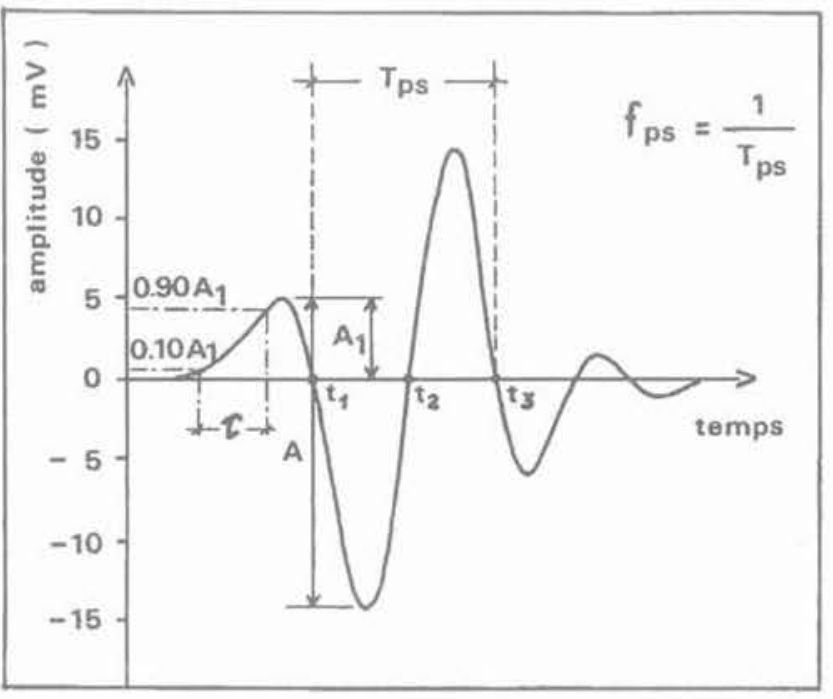

Fig. 3. - Exemple de signal réel (cas du granite de Woas-Wen), définition des paramètres descriptifs $f_{\mathrm{ps}} A_{1}, A$, .

Fig. 3. - Example of a signal: Woas-Wen granite. Definition of the parameters used for describing the signal.

Dans la mesure où l'ensemble du système est linéaire (couplages, électronique, matériau), on peut écrire, dans le domaine fréquentiel:

$$
U(f)=F(f) \times G(f) \times I(f)
$$

avec :

$\mathrm{U}(\mathrm{f})$ : transformée de Fourier du signal recueilli dans l'oscilloscope.

$F(f)=e^{-\alpha(f / L}$ : fonction du transfert du matériau.

La décroissance de l'amplitude d'une onde plane sinusoïdale, au cours de sa propagation, est exponentielle, et définie par le coefficient d'atténuation:

$$
\alpha(f)=\frac{I}{x 2-x 1} \log \frac{A(x 2)}{A(x 1)}
$$

On suppose ici que l'onde progressive, qui parcourt la distance $\mathrm{L}$ dans l'éprouvette, est quasi-plane.
$G(f)$ : fonction de transfert du reste du système; I(f) : impulsion initiale.

Soit $U_{B}$ et $U_{A}$ les signaux correspondant à la roche et à la référence (ici en aluminium); on $\mathrm{a}$ :

$$
\begin{aligned}
\log \left[\frac{U_{R}(f)}{U_{A}(f)}\right]=-\left[\alpha_{R}(f)-\alpha_{A}(f)\right] & \cdot L \\
& +\log \left[\frac{G_{R}(f)}{G_{A}(f)}\right]
\end{aligned}
$$

On fait alors les hypothèses suivantes:

Le coefficient d'atténuation des roches est une fonction linéaire de la fréquence: si besoin est, on restreint l'intervalle de fréquences pour que cette approximation soit valable. Ceci revient à dire que le facteur de qualité:

$$
\mathrm{Q}=\frac{\pi \cdot \mathrm{f}}{\alpha(\mathrm{f}) \cdot \mathrm{V}}
$$

est constant dans l'intervalle considérê.

A titre indicatif, on rappelle que, dans un matériau où $Q=100$ (respectivement : 10), une onde sinusoïdale qui a parcouru une distance égale à une longueur d'onde voit son amplitude multipliée par 0,97 (resp. $0,73)$. Pour laluminium, on admet $Q_{A} \sim \infty$ c'est-àdire $\alpha_{\mathrm{A}} \sim 0$.

. Le terme $\frac{G_{R}(f)}{G_{A}(f)}$ est indépendant de la fréquence, ce qui est inexact en toute rigueur (cf. l'effet de diffraction et celui du couplage échantillon-transducteur, mentionnés au § 2.2.).

Dans ces conditions :

$$
\log \left[\frac{U_{R}(f)}{U_{A}(f)}\right] \sim \frac{-\pi L}{Q_{R} V_{R}} f+K
$$

La figure 4 illustre l'utilisation de cette méthode dans le cas d'un échantillon de marbre de Carrare (ayant subi un chauffage à $150^{\circ} \mathrm{C}$ ): on vérifie que le logarithme du rapport de spectres est une fonction linéaire de la fréquence entre 250 et $800 \mathrm{kHz}$ environ, ce qui permet de calculer par régression linéaire un facteur de qualité. En raison des approximations effectuées, on doit le considérer comme un facteur de qualité «apparent $»$, qui differe d'autant moins du facteur de qualité réel que les impédances acoustiques des roches étudiées sont voisines de celle de la référence.

\section{RÉSULTATS DES MESURES SUR UNE SÉRIE GRANITIQUE À TAILLE DES GRAINS CROISSANTE}

\subsection{Choix des matériaux}

On a choisi six roches granitiques de Bretagne et des Vosges, dont les principales caractéristiques sont données dans le tableau $\mathrm{L}$. 


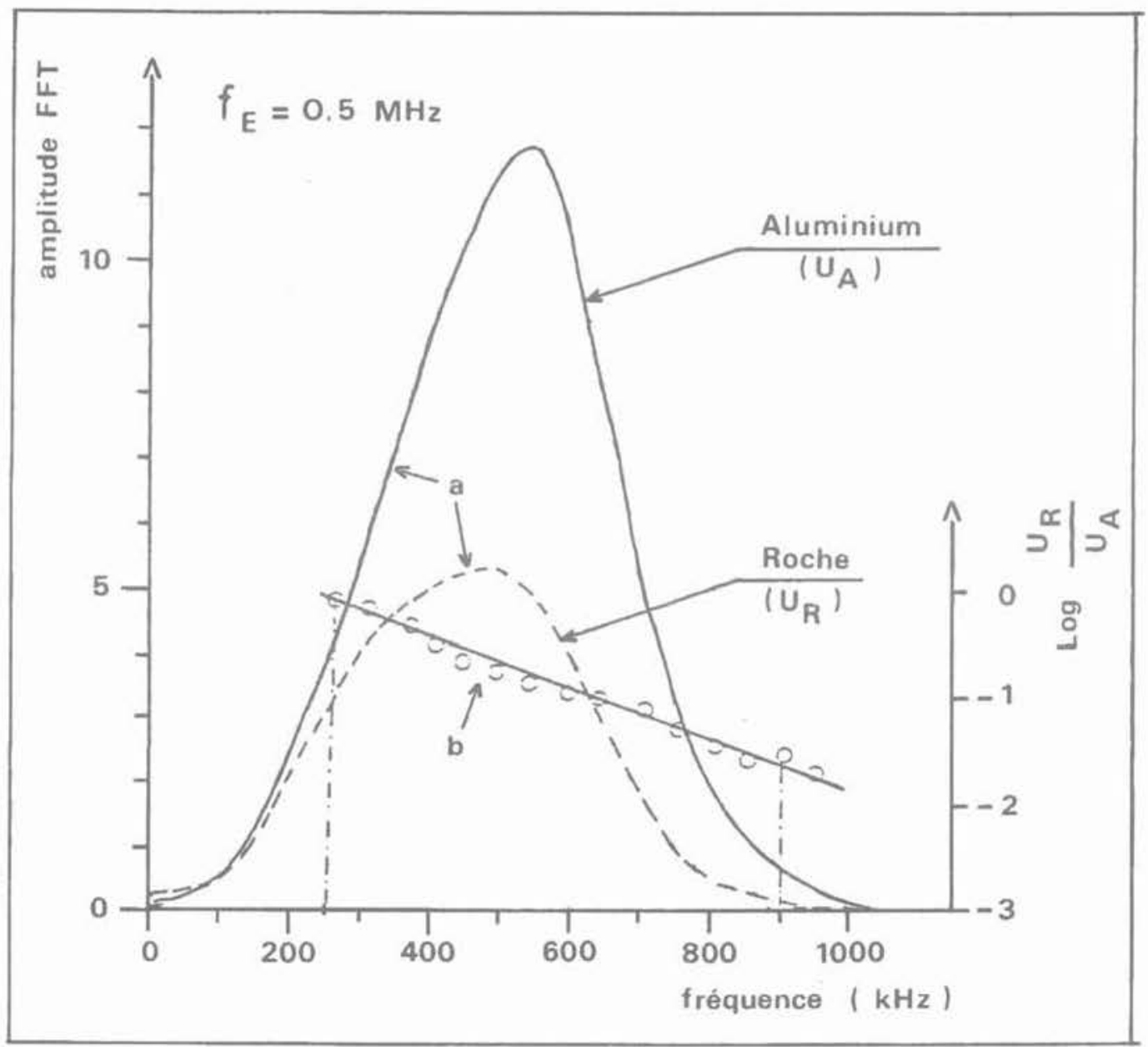

Fig. 4. - Evaluation du facteur de qualité par la méthode du rapport de spectres: exemple d'un marbre de Carrare préfissuré par chauffage à $150^{\circ} \mathrm{C}$; a: modules des transformées de Fourier des signaux de la roche et de l'aluminium; b: rapport des spectres. La pente de la droite, ajustée par moindres carrés $(r=0,992)$, est égale à -0.002540 , ce qui correspond à $Q \mathrm{~V}=81640 \mathrm{~m} / \mathrm{s}$, d'où $Q=28$.

Fig. 4. - Fourier spectral ratio of the Carrare marble (reference of Aluminium) : a: Fourier spectra of rock and of reference; $b$; spectral ratio (the calculated quality factor is $0 \simeq 28$ ).

Tableau I. Principales caractéristiques des granites étudiés

\begin{tabular}{|c|c|c|c|c|c|c|}
\hline & $\begin{array}{l}\text { Densité } \\
\text { réelle }\end{array}$ & $\begin{array}{l}\text { Vitesse } \\
\text { longitud. }\end{array}$ & $\begin{array}{l}\text { Impédance } \\
\text { acoustique }\end{array}$ & $\begin{array}{l}\text { Indice } \\
\text { de } \\
\text { continuité }\end{array}$ & $\begin{array}{l}\text { Résist. } \\
\text { en compress. }\end{array}$ & $\begin{array}{c}\text { Taille } \\
\text { des } \\
\text { grains }\end{array}$ \\
\hline Echantillon & $\left(\mathrm{kg} / \mathrm{m}^{3}\right)$ & $\underset{(\mathrm{m} / \mathrm{s})}{\mathrm{V}_{\mathrm{p}}}$ & $\begin{array}{c}Z=p \cdot V_{p} \\
\left(\mathrm{~kg} \cdot \mathrm{m}^{-2} \cdot \mathrm{s}^{-1}\right)\end{array}$ & $\begin{array}{r}\text { I.C. } \\
\%\end{array}$ & $\begin{array}{c}\mathrm{R}_{\mathrm{c}} \\
(\mathrm{MPa})\end{array}$ & $\stackrel{\varnothing}{(\mathrm{mm})}$ \\
\hline APL & 2624 & 5200 & $13,64 \times 10^{6}$ & 89 & 375 & 0,1 \\
\hline VI & 2713 & 5250 & $14,24 \times 10^{6}$ & 89 & 192 & 0,9 \\
\hline KR & 2612 & 4600 & $12,02 \times 10^{6}$ & 77 & 227 & 1,2 \\
\hline WW & 2586 & 4200 & $10,86 \times 10^{6}$ & 70 & 233 & 2,0 \\
\hline LH & 2647 & 4400 & $11,65 \times 10^{6}$ & 74 & 182 & 3,0 \\
\hline SN & 2671 & 5200 & $13,89 \times 10^{6}$ & 88 & 184 & 4,5 \\
\hline
\end{tabular}




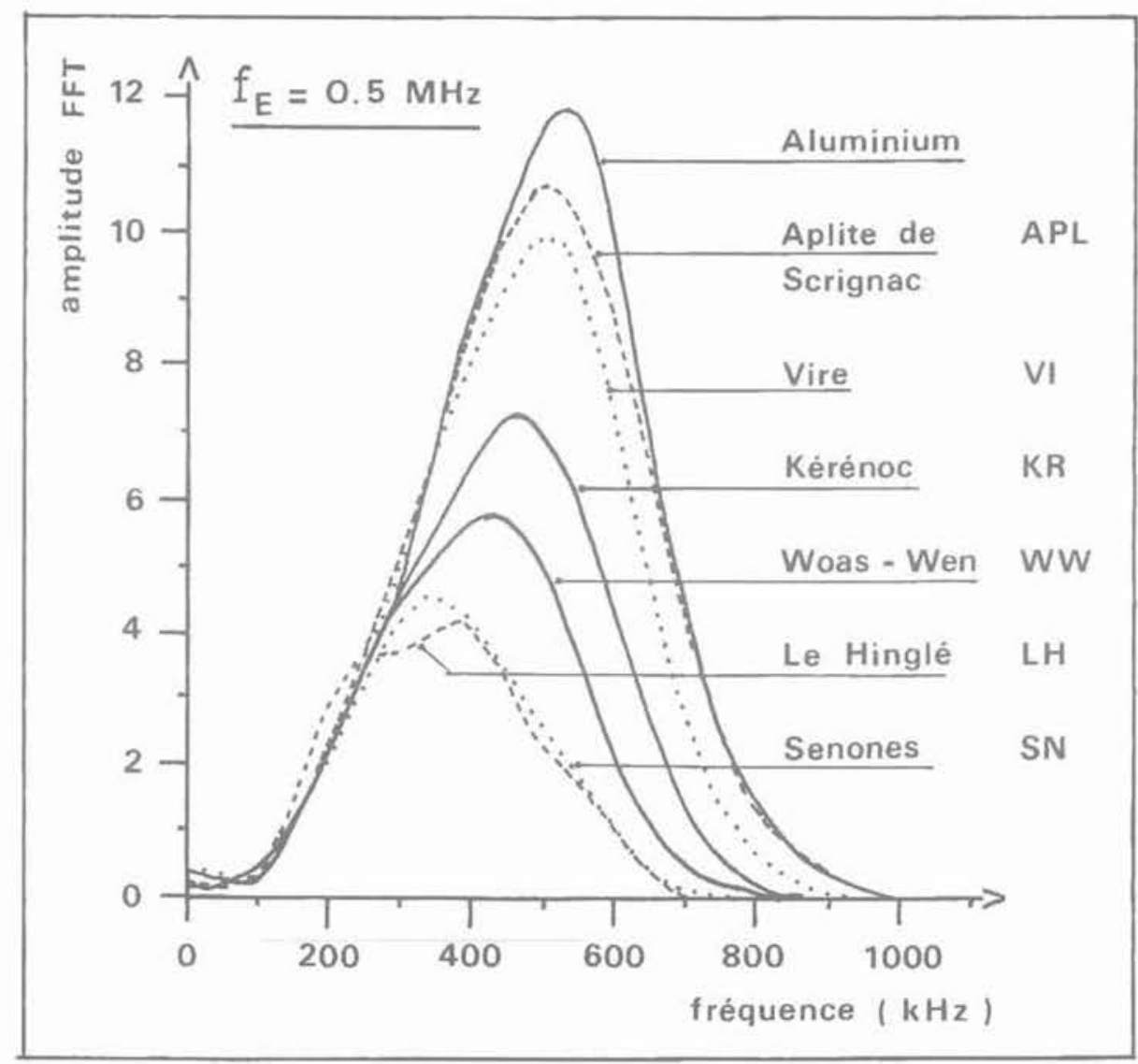

Fig. 5. - Modules des transformées de Fourier (F.F.T.) du signal obtenu avec les transducteurs $0,5 \mathrm{MHz}$ : série granitique.

Fig. 5. - Fourier amplitude spectra (F.F.T.): granites

of different grain sizes, with $0.5 \mathrm{MHz}$ transducers.

On a travaillé sur des matériaux à minéralogie à peu près identique et à degré d'altération comparable, La dernière exigence n'est pas toujours respectée, ce qui a conduit à quelques $*$ anomalies $»$ qu'on commentera par la suite.

\subsection{Les résultats}

On présente les résultats obtenus par la méthode du rapport des spectres.

La figure 5 illustre bien linfluence de la taille des grains dans le domaine fréquentiel du signal.

L'examen des différents paramètres du signal dans les domaines temporel et fréquentiel conduit à des corrélations intéressantes, telles que celle présentée dans la figure 6 .

\subsection{Discussion}

$\mathrm{Si}$ on s'intéresse à la mesure de l'atténuation, les amplitudes du signal sont des paramètres mal adaptés, à cause de leur dépendance vis-à-vis des propriétés élastiques du matériau. Dans le cas de la série grani- tique traitée, ce problème se pose peu ou pas, puisque les impédances acoustiques des granites testés sont peu différentes (voir tableau I).

L'interprétation de ces résultats repose sur les phéno. mènes d'interaction entre l'onde et l'agrégat polycristallin que constitue la roche. Le terme de diffraction est alors très généralement employé pour désigner cette interaction, tant que la longueur d'onde ( $\lambda$ ) est grande devant la dimension moyenne des grains $(\varnothing)$, ou du même ordre de grandeur. Dans le premier cas, on peut rechercher un milieu homogène atténuant équivalent; dans le deuxième cas, l'onde est en fait soumise à une succession de réflexions et réfraction sur les joints de grains : on parlera de diffraction stochastique pour désigner l'effet macroscopique résultant.

Des calculs théoriques approchés (MERKULOV, 1956 ; PAPADAKIS, 1965) fournissent les résultats suivants :

- dans le cas de l'approximation de Rayleigh, valable pour $\lambda \gg \varnothing$, le coefficient d'atténuation $\alpha$ varie comme $\varnothing^{3} \times f^{4}: Q$ varie comme $(\lambda / \varnothing)^{3}$;

- dans le cas $\lambda \sim \varnothing$ (en pratique, pour $\lambda<10 \varnothing)$. on aurait une dépendance de $\alpha$ en $\varnothing \times \mathrm{f}^{2}$ (ou de $\mathrm{Q}$ en $\lambda / \varnothing)$. 


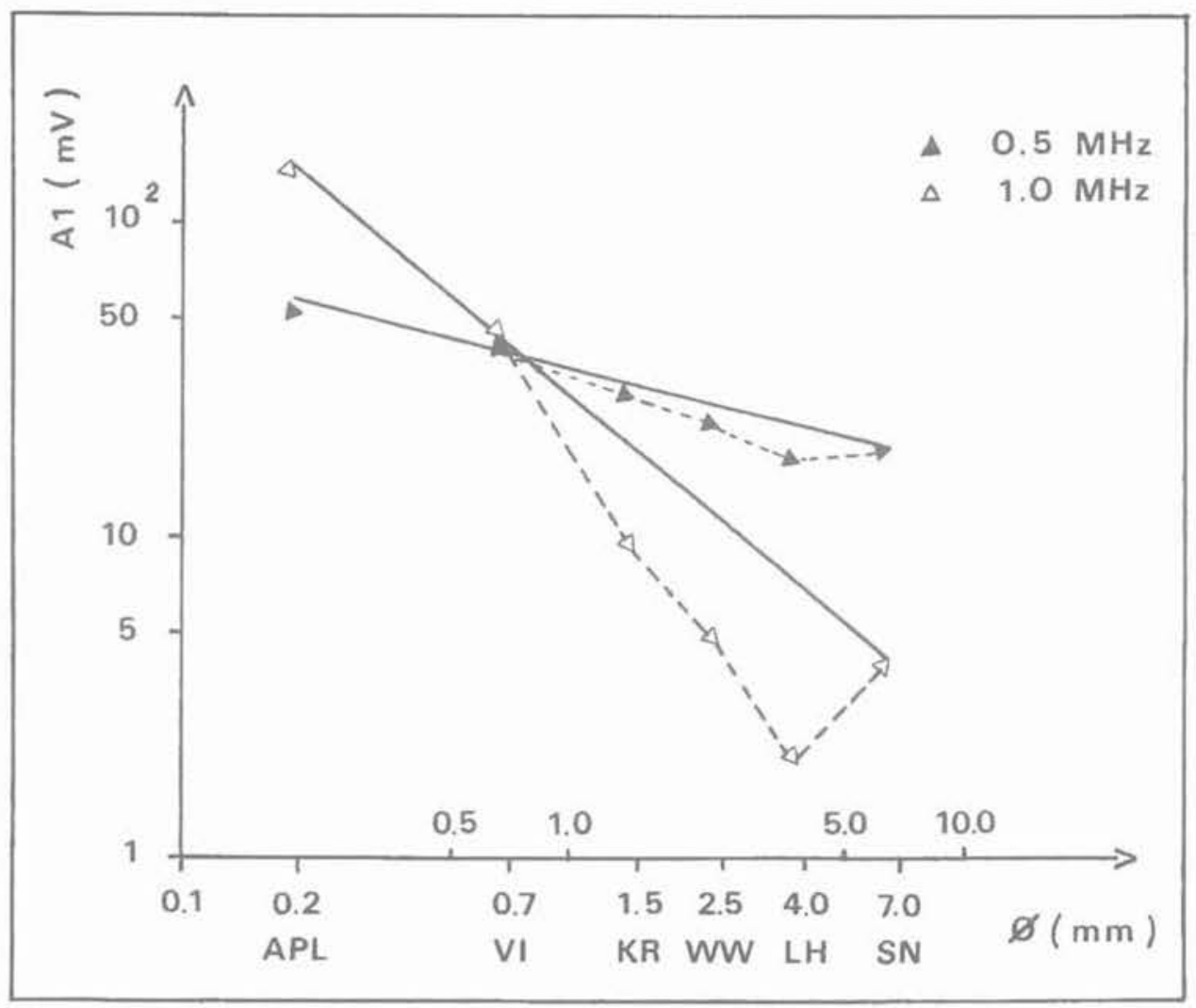

Fig. 6. - Relation entre la première amplitude $\left(A_{1}\right)$ du signal temporel et la taille moyenne des grains ( $\left.\varnothing\right)$ d'une série granitique à texture différente (fréquences centrales: 0,5 et $1,0 \mathrm{MHz}$ ).

Fig. 6. - First amplitude of the signal as a function of grain size (Ø). Granitic rocks, with 0.5 and 1.0 MHz transducers

Par ailleurs, les études expérimentales de WINKLER (1983) sur des grès poreux ont mis en évidence une variation de $\alpha$ en $\mathrm{fm}^{\mathrm{m}}, 3<\mathrm{m}<4$, et ce pour des valeurs de $N / \varnothing$ entre 5 et 25 .

Dans nos expériences, le rapport $\frac{\lambda}{\varnothing}$ varie essentielle

ment de 1 (cas du granite SN) à 130 (cas de l'aplite $\mathrm{APL}$ ) : on vérifie (figure 7) que l'atténuation est d'autant plus forte que la longueur d'onde est petite et que la taille des grains est grande, c'est-à-dire que $\lambda / \varnothing$ se rapproche de 1. Pour les granites VI, KR, WW, $\mathrm{LH}$ et $\mathrm{SN}$, le coefficient d'atténuation varie comme $p-2,7 \leqslant p \leqslant 3,5-$.

Ce résultat paraît raisonnable étant donné que d'une part l'approximation de Rayleigh n'est pas strictement $(1<\lambda / \varnothing<10)$ respectée pour ces granites et que d'autre part $\varnothing$ désigne en fait un diamètre moyen qui représente toute une "granulométrie » de cristaux.

En ce qui concerne l'aplite $\left(\frac{\lambda}{\varnothing}\right.$ vaut 130 à $1 \mathrm{MHz}$ et

65 à $0,5 \mathrm{MHz}$ ), les prévisions théoriques en approximation de Rayleigh ne correspondent ni en valeur, ni en dépendance fréquentielle, avec nos résultats expérimentaux. L'aplite apparaît ici comme assez faiblement atténuante, suivant un mécanisme mal identifié, où $Q$ est indépendant de la fréquence, et qui masquerait l'influence bien plus faible de la diffraction. Il est possible aussi que la chaîne expérimentale et la méthode utili-

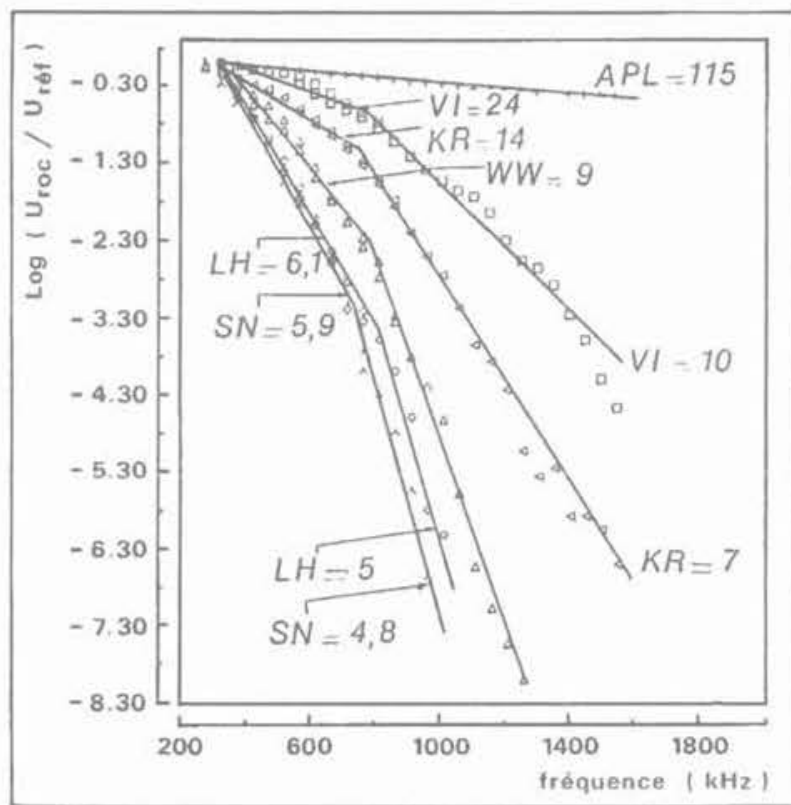

Fig. 7. - Série granitique: rapports de spectres correspondant aux transducteurs 0,5 et $1,0 \mathrm{MHz}$. On a calculé les facteurs de qualité pour chacune de ces fréquences: les rapports de spectres ont été décomposés en deux segments de droite adjacents.

Fig. 7. - Fourier spectral ratios 10.5 and $1.0 \mathrm{MHz}$ transducers) of the granitic rocks. Two quality factors were calculated for each rock, corresponding to different frequency domains. 
sées ne soient guère capables de différencier des atté. nuations faibles $(Q>150$ ?).

Un autre point intéressant à commenter est « l'anomalie * du granite de Sénones (S.N.). Cette " anomalie » est observée dans la corrélation $(A, \varnothing)$ de la figure 6 . On constate en particulier que les trois granites K.R., W.W. et L.H. sont décalés vers le bas par rapport au reste des granites. Cette distinction s'explique par la différence de lindice de continuité entre ces deux groupes. On enregistre par conséquent deux phéno. mènes imbriqués: d'une part l'atténuation due à la diffraction sur grains et d'autre part l'atténuation due à l'altération.

\section{RÉSULTATS DES MESURES SUR UNE SÉRIE DE GRANITES DE PLUS EN PLUS ALTÉRÉS}

\subsection{Le matériau}

L'aplite de Scrignac est une roche granitique à structure très homogène et à grains très fins $(0,08 \mathrm{~mm})$. Il s'agit d'une roche très compacte, ceci étant vérifié par ses qualités mécaniques. Cette roche est essentiellement composée de quartz et de feldspaths parfois séricitisés; on trouve également de la muscovite.

On a choisi trois états d'altération de plus en plus poussée, sur lesquels les résultats suivants ont été obtenus:
On obtiendrait les trois valeurs ci-dessous, à comparer à l'indice de continuité:

\begin{tabular}{|l|c|c|c|}
\hline & APL $_{\text {i }}$ & APL & APL \\
\hline I.A. (\%) & 100 & 35 & 25 \\
I.C. (\%) & 89 & 76 & 39 \\
\hline
\end{tabular}

b. Les rapports de spectres présentés dans la figure 8 admettent une approximation linéaire correcte dans la plage de fréquences examinée : 250 à $3200 \mathrm{kHz}$.

De l'état I à l'état II, l'altération se manifeste par une augmentation de la porosité totale due quasi exclusivement à un accroissement du réseau de microfissures, parfois remplies de produits d'altération et par une détérioration de l'état des minéraux (feldspaths). Le passage de l'état II à l'état III est caractérisé par une dégradation complète des feldspaths (souvent trans. formés en un agrégat cryptocristallin), parfois par des dissolutions partielles des minéraux et par un réseau de microfissures plus dense. Les vides ainsi créés peuvent être assimilés à des discontinuités de type pore.

Le facteur de qualité $(\mathrm{Q})$ calculé à partir de la figure 8 se montre constant et par conséquent les mécanismes de diffraction sur les hétérogénéités (grains, pores et fissures) ne semblent pas convenir comme interprétation satisfaisante de l'atténuation mesurée. Il faudrait donc faire appel à des mécanismes à $\mathrm{Q}$ constant, dont l'origine reste plutôt mal connue; les minéraux argileux avec molécules d'eau adsorbées (même à l'état $\propto \mathrm{sec}$ »

Tableau II. Caractéristiques d'une aplite dans trois états d'altération

\begin{tabular}{|c|c|c|c|c|c|}
\hline Aplite & $\begin{array}{c}\text { Porosité } \\
\mathrm{n} \%\end{array}$ & $\begin{array}{c}\text { Masse volumique } \\
\text { réelle } \\
\left(\mathrm{kg} / \mathrm{m}^{3}\right)\end{array}$ & $\begin{array}{c}\text { Vitesse } \\
\text { longitud. } \\
\mathrm{V}_{\mathrm{p}}(\mathrm{m} / \mathrm{s})\end{array}$ & $\begin{array}{c}\text { Indice de } \\
\text { continuité } \\
\text { I.C. (\%) }\end{array}$ & $\begin{array}{c}\text { Résistance en } \\
\text { compres. simple } \\
(\mathrm{MPa})\end{array}$ \\
\hline I & 0,86 & 2624 & 5200 & 89 & 353 \\
\hline II & 6,6 & 2495 & 4100 & 76 & 169 \\
\hline III & 11,9 & 2358 & 2350 & 39 & 60 \\
\hline
\end{tabular}

\subsection{Résultats et commentaires}

5.2.1. Les résultats obtenus par la méthode du rapport des spectres sont illustrés sur la figure 8 dans le cas des trois fréquences centrales: $0,5 \cdot 1,0 \cdot 2,25 \mathrm{MHz}$, balayant ainsi une plage de $250 \mathrm{kHz}$ à $3200 \mathrm{kHz}$.

Deux points principaux se dégagent à partir de la figure 8:

a. Le facteur de qualité (Q), tout comme lindice de continuité est très sensible à l'état d'altération du matériau; il semble être plus sensible à un début d'altération que l'indice de continuité.

On pourrait proposer un « indice d'altération *:

$$
\mathrm{L} A \mathrm{.}=\frac{\mathrm{Q} \text { (roche) }}{\mathrm{Q} \text { (mêrne roche à l'état sain) }}
$$

en laboratoire) jouent probablement un rôle dans ces mécanismes.

5.2.2. Il ressort des mesures de vitesse et d'atténuation que le paramètre Q.V est un indicateur particulièrement discriminateur de l'état d'altération. D'après GLADWIN et STACEY (1974), on a, entre ce paramètre et le temps de montée $\tau$, la relation empirique $\tau=\tau_{0}+C \frac{L}{Q . V_{p}}$, où $\tau_{0}$ et $C$ sont des constantes dépendant de la source.

La figure 9 confirme la validité d'une telle relation pour chacun des couples de transducteurs; bien entendu, il faudrait multiplier les points expérimentaux pour pouvoir établir une loi générale. 


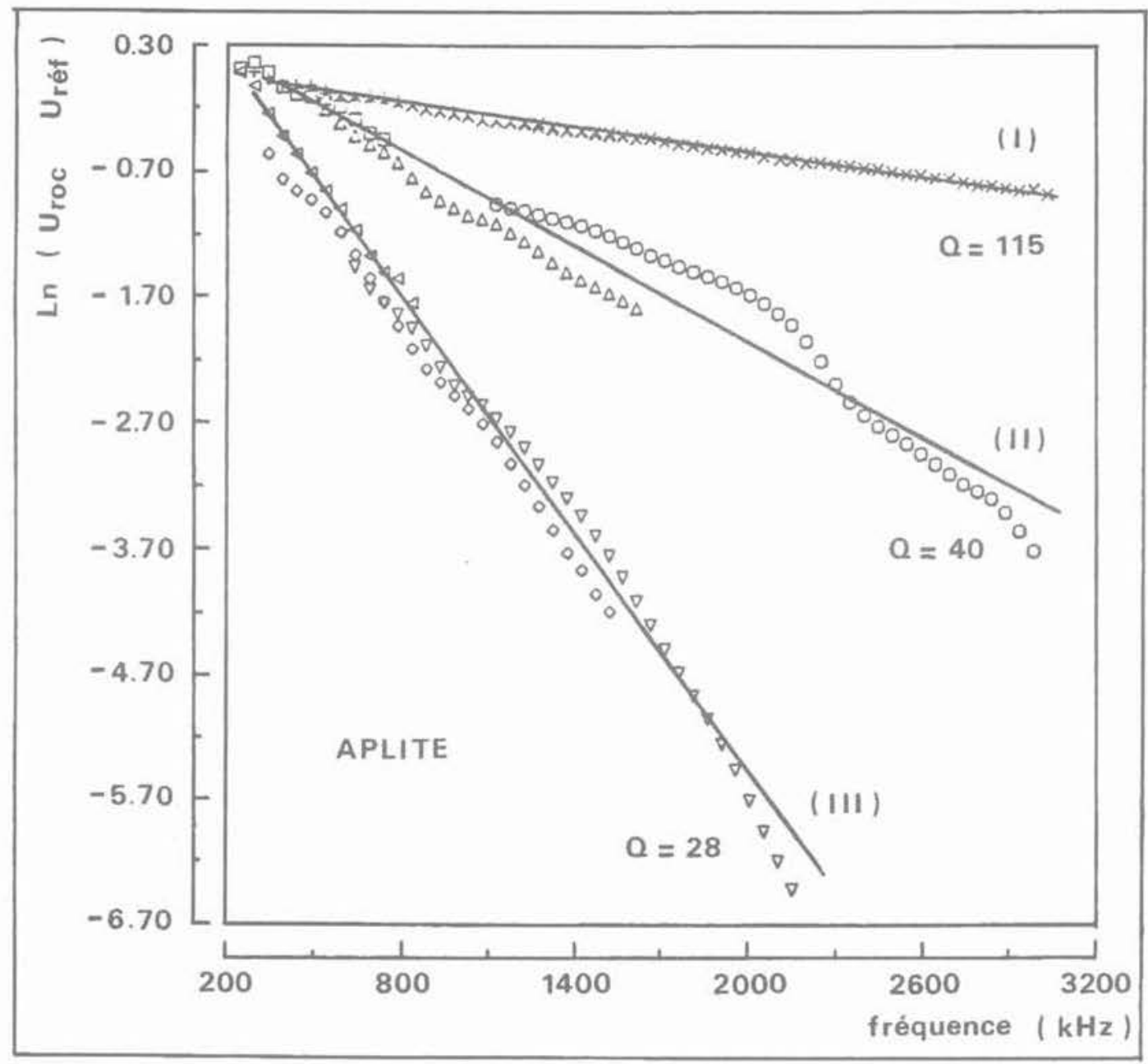

Fig. 8. - Rapport des spectres pour une aplite de plus en plus altérée (3 états d'altération : I - II - III) aux trois fréquences centrales de $0,5-1,0$ et $2,25 \mathrm{MHz}$.

Fig. 8. - Fourier spectral ratios of the same aplitic rock in three weathering states (I - II - III). Three pairs of transducers $(0.5-1.0-2.25 \mathrm{MHz})$ were used.

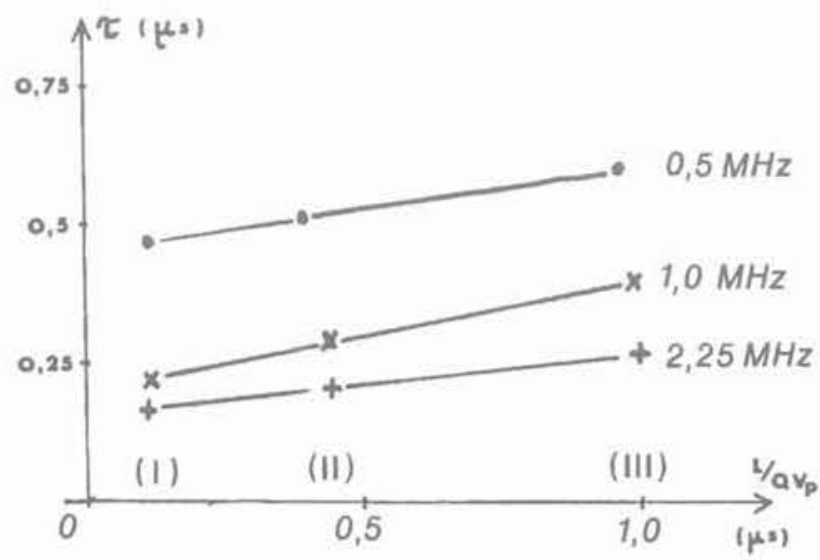

Fig. 9. - Relation linéaire entre le temps de montée ( $)$ et le rapport $\left(L / Q . V_{p}\right)$ pour les trois fréquences centrales: 0,5 - 1,0 et $2,25 \mathrm{MHz}$ dans le cas d'une aplite de plus en plus altérée (trois états d'altération: I - II - III).

Fig. 9. - Rise time as a linear function of $L / Q \times V_{D}$ ratio, for the aplitic rock in three weathering states.
On pourrait donc proposer que la mesure du temps de montée $\tau$, plus facile à pratiquer que le rapport des spectres, serve à déterminer le paramètre Q.V (cependant la pente, faible, des droites obtenues figure 9 n'est pas très favorable).

\section{CONCLUSION}

En définitive, on dispose d'un essai fiable, permettant d'évaluer les propriétés atténuantes d'une roche sèche; il s'agit toutefois d'un essai sophistiqué, nécessitant des conditions expérimentales rigoureuses, donc bien différent de la simple mesure de la vitesse.

L'atténuation est fonction de la taille des grains, lorsque la longueur d'onde devient du même ordre de grandeur que ceux-ci. On a observé que le coefficient d'atténuation augmentait à peu près comme la troisième puissance de la fréquence, en liaison avec un phénomène 
de diffraction sur les grains. Pour les roches à grain fin, le coefficient d'atténuation varie linéairement avec la fréquence.

L'atténuation est très sensible au degré d'altération d'un matériau (ici une aplite). Le produit $\mathrm{Q} \times \mathrm{V}$ (facteur de qualité $\times$ vitesse longitudinale) devrait permettre de déceler de faibles différences d'altération et il peut être estimé à l'aide du paramètre temps de montée.

L'essai décrit ci-dessus, offre dans beaucoup de cas une information redondante vis-à-vis de l'indice de continuité, issu d'une simple mesure de vitesse; mais la mesure de l'atténuation peut constituer un outil d'identification des roches intéressant, puisqu'il apporte des précisions concernant la structure des roches (taille des grains, et, plus généralement, des hétérogénéités telles que pores, fissures ou débris fossiles) et leur état (altération par exemple).

\section{BIBLIOGRAPHIE}

BOURBIÉ, COUSSY, ZINSZNER (1986), Acoustique des milieux poreux, Editions Technip. 339 pages.

GLADWIN, STACEY (1974), Anelastic degradation of acoustic pulses in rock, Phys. Earth Plan. Int., 8, 332-336.
KLIMIS (1987), Etude en laboratoire de l'atténuation des ondes longitudinales: application à la caracté. risation géotechnique des roches, Thèse présentée à l'Ecole Nationale supérieure des Mines de Paris (9 décembre 1987).

MERKULOV (1956), Investigation of ultrasonic scattering in metals, Soviet. Phys. Techn. Phys., vol. 1, pp. 59-69.

PAPADAKIS (1965), Revised Grain-Scattering Formulas and Tables, J. Acoust. Soc. Am., 37, 4, pp. 703710.

TOKSÖZ, JOHNSTON, TIMUR (1979), Attenuation of seismic waves in dry and saturated rocks: 1. Laboratory measurements, Geophysics, vol. 44, $\mathrm{n}^{\circ} 4$, pp. $681-690$.

TOURENQ, FOURMAINTRAUX, DENIS (1971), Propagation des ondes et discontinuités des roches, Symposium Soc. Intern. Mécanique des Roches, Nancy.

WINKLER (1983), Frequency dependent ultrasonic properties of high porosity sandstone, J. Geophys. Res., 88, pp. 9493-9499.

WINKLER, PLONA (1982), Technique for measuring ultrasonic velocity and attenuation spectra in rocks under pressure, J. Geoph. Res. vol. 87, B13, pp. 10776-10780. 